\title{
Skeletal Reaction Model Generation, Uncertainty Quantification and Minimization: Combustion of Butane
}

\author{
Y.X. Xin ${ }^{1}$, D. A. Sheen ${ }^{2}$, H. Wang ${ }^{3, *}$, C.K. Law ${ }^{1}$ \\ ${ }^{1}$ Department of Mechanical and Aerospace Engineering, Princeton University, Princeton, NJ \\ 08544, USA \\ ${ }^{2}$ Chemical Sciences Division, National Institute of Standards and Technology \\ Gaithersburg, MD 20899, USA \\ ${ }^{3}$ Department of Mechanical Engineering, Stanford University, Stanford, CA 94305, USA
}

\begin{abstract}
Skeletal reaction models for $n$-butane and iso-butane combustion are derived from a detailed chemistry model through directed relation graph (DRG) and DRG-aided sensitivity analysis (DRGASA) methods. It is shown that the accuracy of the reduced models can be improved by optimization through the method of uncertainty minimization by polynomial chaos expansion (MUM-PCE). The dependence of model uncertainty on the model size is also investigated by exploring skeletal models containing different number of species. It is shown that the dependence of model uncertainty is subject to the completeness of the model. In principle, for a specific simulation the uncertainty of a complete model, which includes all reactions important to its prediction, is convergent with respect to the model size, while the uncertainty calculated with an incomplete model may display unpredictable correlation with the model size.
\end{abstract}

Keyword: model reduction, model optimization, uncertainty quantification

* Corresponding author:_haiwang@stanford.edu 


\section{Introduction}

Detailed kinetic modeling is a useful tool to probe intricate fuel combustion and flame phenomena. The modeling approach usually involves the use of a detailed reaction model consisting of the relevant chemical species and elementary reactions that may participate in sequential and parallel kinetic processes of fuel oxidation. In such a model, the rate parameters are usually derived from experimental rate measurements, reaction rate theories, or, in many cases, estimations from analogous reactions. In all cases, the values of the rate parameters can be uncertain to various extents [1]. Consequently the uncertainty of the model parameters causes the prediction to be correspondingly uncertain —an issue that has received an increased attention and has been discussed in a variety of context in recent years (see, e.g., [2-25]).

Recent efforts directed at developing reaction models for practical liquid fuels and their surrogates have led to the emergence of large models, some of them may contain $\mathrm{O}\left(10^{3-4}\right)$ species and $\mathrm{O}\left(10^{4-5}\right)$ reactions (see, e.g., [26]). The use of these detailed models in computational fluid dynamics (CFD) introduces another problem. That is, they are too large to be practical for CFD applications and must be reduced in size and complexity before they can be used [27, 28]. Consequently, various model reduction strategies have been proposed (see, e.g., [28-30]). In general, the methods of model reduction may be categorized into skeletal reduction and time scale analysis. Skeletal reduction eliminates unimportant species and reactions, which can be achieved by sensitivity analysis [30-33], principal component analysis [34], Jacobian analysis [32], optimization [3], detailed reduction [35], directed relation graph (DRG) ([36-38]), DRG with error propagation [39], and DRG-aided sensitivity analysis (DRGASA) [40, 41]. Time-scale analysis identifies fast species as well as reactions, and describes their time evolutions by algebraic equations. Methods of time-scale analysis are primarily based on quasi-steady-state (QSS) and partial equilibrium (PE) assumptions [42-46]. Several approaches to identifying the 
QSS species have been proposed [45-52]. Other, and perhaps more systematic approaches to time-scale analysis include intrinsic low-dimensional manifolds (ILDM) [53] and computational singular perturbation (CSP) [54-57].

In the present study, we propose an improved model reduction strategy by integrating the processes of skeletal reduction with model optimization and uncertainty quantification. In this strategy, a skeletal model is derived using DRGASA. The rate coefficients in skeletal models are then subject to a multi-parameter constraining against full-model predictions of a prescribed set of combustion properties, so that the model can be made predictive when DRGASA is used in an aggressive manner to generate ever-smaller models. We also explore the correlation between model size and the uncertainty in its predictions in order to identify when models are reduced beyond any capacity to be predictive. The paper is organized as follows. In Section 2, the research strategies of model skeletal reduction, optimization and uncertainty quantification, are specified in detail. In section 3, models of different sizes were generated from a detailed chemistry model, USC Mech II, [58], using $n$-butane and $i$-butane combustion as examples. The uncertainties of the skeletal models are quantified by the spectral expansion technique [20] and correlated to model size. Conclusions are summarized in Section 4.

\section{Methodology}

\subsection{Skeletal Reduction}

A detailed reaction model is composed of three types of species: critical ones, nonessential ones, and marginal ones. For a given simulation, critical species participate in those reaction channels that largely determine the simulation results, while nonessential species only participate in reaction channels having little to no influence on the simulation results. The categorization of any particular species is conditional, subject to the specific simulation circumstance. A successful skeletal reduction retains all critical species, eliminates all nonessential species, and 
properly deals with the marginal ones. The importance of species can be assessed by two types of method, examining the species conversion flux (e.g., [36-39]) or sensitivity analysis [30-32, 41]. These two methods have their respective advantages and disadvantages. Flux analysis is affordable computationally and able to identify nonessential species efficiently. However, it can misestimate the importance of marginal and critical species. Sensitivity analysis quantifies the importance of species with better accuracy but it often requires prohibitive computational costs, hence preventing its application in reducing large models. Here, the skeletal model reduction adopts a two-stage procedure [41]: the detailed model is first reduced by flux analysis to an intermediate-size model from which a final skeletal model is generated by sensitivity analysis. In the present study, this two-stage strategy is conducted by combining DRG with DRGASA.

\subsubsection{Overview}

Model reduction always requires that the conditions be defined against which the model will be reduced. In this work, we developed a reduced model for the oxidation of butane. As such, the model was reduced using a set of laminar flame and auto-ignitions of butane/air mixtures. The conditions considered are listed in Table 1 and cover pressure in the range of 1 atm $\leq p \leq 20$ atm, equivalence ratio $0.6 \leq \phi \leq 1.5$, initial temperature $800 \mathrm{~K} \leq T_{0} \leq 1500 \mathrm{~K}$ for auto-ignition, and inlet temperature $T_{i n}=300 \mathrm{~K}$ for laminar flame speed. There are a total of 45 ignition delay times and 9 flame speeds. The auto-ignition and flame propagation of $n$-butane-air and $\underline{i}$-butane-air mixtures were simulated using USC Mech II [58]. The model was developed for high-temperature oxidation of $\mathrm{H}_{2}, \mathrm{CO}$ and $\mathrm{C}_{1}-\mathrm{C}_{4}$ hydrocarbons and consists of 111 species and 784 reactions.

\subsubsection{Directed Relation Graph Model Reduction}

Both DRG and DRGASA have been discussed in detail elsewhere [36-38, 41], and as such are presented here only briefly. DRG represents a chemistry model by a directed relation graph, whose nodes are species and the width of the edge from node $m$ to $n, r_{m n}$, is calculated as: 


$$
r_{m n}=\frac{\sum_{i=1, N_{R}}\left|v_{i, m} \omega_{i} \delta_{n}^{i}\right|}{\sum_{i=1, N_{R}}\left|v_{i, m} \omega_{i}\right|}
$$

where

$$
\delta_{n}^{i}=\left\{\begin{array}{cc}
1, & \text { if the } i \text {-th reaction involves species } n \\
0, & \text { otherwise }
\end{array}\right.
$$

and the subscripts $m$ and $n$ identify the species. Additionally, $N_{R}$ is the total number of reactions, $v_{i, m}$ the stoichiometric coefficient of species $m$ in the $i^{\text {th }}$ reaction, and $\omega_{i}$ the net reaction rate of the $i^{\text {th }}$ reaction. The value of $r_{m n}$ quantifies the importance of species $n$ to the production of species $m$. For a given user-specified threshold $\varepsilon$, skeletal reduction is conducted by truncating edges narrower than $\varepsilon$. A species is eliminated when all edges linking to it are removed during this process. With increasing $\varepsilon$, an increased number of species is removed. The critical $\varepsilon$ value, corresponding to the elimination of a certain species, is utilized as the DRG index of this species. An intermediate-size skeletal model is generated by removing species with a small DRG index, typically less than 0.1 , and the remaining species are further examined in the subsequent sensitivity analysis through DRGASA. It should be noted that, since there are many conditions against which the model is reduced, the overall DRG index for a given species is defined to be the maximum DRG index across all conditions, so that if a species must be kept to reproduce one condition, it is kept for all conditions; in other words, the skeletal model reduced against all conditions is the union of skeletal models reduced for each condition. 


\subsubsection{Directed Relation Graph-Aided Sensitivity Analysis}

It is possible that DRG will result in the removal of relatively few species from the model, which would not be an especially useful model reduction technique. USC Mech II is an example of such a model, as will be shown in Section 3. As a result, the DRGASA method [40, 41] was developed to enable a more aggressive reduction of the skeletal models. In DRGASA, species with a large DRG index, chosen to be greater than 0.5 in this work, are defined to be critical and kept in the final skeletal model without sensitivity test. What remains, then is to sort the remaining species into the marginal and nonessential sets. To accomplish this, the importance of a particular species is defined as the maximum relative error, err $_{\text {max }}$, in a model prediction caused by the removal of that species. In this case, each reduction condition has the reference value $\eta_{r}^{\text {ref }}$ predicted by the skeletal model. When species $k$ is removed from the skeletal model, there is a new predicted value $\eta_{r, k}^{*}$. There is then an error for each species, $\operatorname{err} r \cdot k=\left(\eta_{r}^{\text {ref }}-\eta_{r, k}^{*}\right) / \eta_{r}^{\text {ref }}$, and $e r r_{\max }$ for a species is the maximum, over all conditions $r$, of the $e r r_{r, k}$. The examined species are ranked by their importance, and model reduction is carried out by successively eliminating species of the least importance, until the resulting model cannot satisfy a prescribed accuracy requirement.

\subsection{Model Optimization and Uncertainty Quantification}

Removal of reaction pathways can lead to quantitative changes in the model prediction. Because the dimensionality of the reduced model generally remains large, it should be possible to constrain the reduced model against the detailed model by an error minimization procedure. Rate coefficients subject to the minimization procedure are selected using a one-at-a-time sensitivity analysis [53]. For each computational experiment the uncertainty-weighted sensitivity coefficient $S_{r, i}$ is defined as,

$$
S_{r, i}=\frac{d \ln \eta_{r}}{d \ln A_{i}} \ln f_{i}
$$


where $\eta_{r}$ is the response, $A_{i}$ and $f_{i}$ are the Arrhenius pre-exponential factor and the uncertainty factor of the $i^{\text {th }}$ rate parameter, respectively. The active rate parameters are selected here as those with $S_{r, i}>\max \left(S_{r, i}\right) / 10$.

For illustration purposes, the responses selected here are ignition delay time and laminar flame speed. Ignition delay time simulations were performed using a kinetic integrator based on the DVODE solver [59] and the chemical production terms provided by ChemKin II [60]. Laminar flame speed was computed using Sandia PREMIX [61] and TRANSPORT [62] with modifications from [63].

MUM-PCE has been detailed elsewhere [20] and is summarized here. It is assumed that the uncertain parameters in the model can be expressed using the first-order polynomial chaos expansion (PCE) $\mathbf{X}=\mathbf{x}^{(0)}+\mathbf{x}^{(1)} \boldsymbol{\xi}$, where $\mathbf{x}^{(0)}$ is the reduced parameter vector whose elements are

$$
x_{i}^{(0)}=\frac{\ln \left(\theta_{i} / \theta_{i, 0}\right)}{\ln f_{i}}
$$

where $\theta_{i}$ is the $i^{\text {th }}$ uncertain parameter and $\theta_{i, 0}$ its nominal value. $\xi$ is a vector of independent, identically distributed (i.i.d) normal random variables with mean 0 and variance $1 . \mathbf{x}^{(1)}$ is a transformation matrix, so that $\mathbf{X}$ follows a multivariate normal distribution with mean $\mathbf{x}^{(0)}$ and covariance matrix $\Sigma=\mathbf{x}^{(1)} \mathbf{x}^{(1) T}$.

MUM-PCE applies Bayes' Theorem to determine the joint probability density function (PDF) of the reduced parameters, which results in the following PDF for the constrained rate parameters,

$$
\ln P_{\mathbf{X}}(\mathbf{x}) \sim-\left[\sum_{r=1}^{N_{e}}\left(\frac{\eta_{r}(\mathbf{x})-\eta_{r}^{\mathrm{det}}}{\sigma_{r}^{\mathrm{tol}}}\right)^{2}+4 \sum_{i=1}^{N_{r}} x_{i}^{2}\right]
$$


The second term in right-hand side of Eq. 4 represents the prior information coming from the uncertainty estimates of the reduced model, while the first term contains additional information from requiring that the reduced model reproduce the behavior of the detailed model. Here, $\eta_{r}(\mathbf{x})$ is the reduced model prediction expressed as a function of the rate parameters $\mathbf{x}, N_{e}$ the number of computational experiments, $N_{r}$ the number of active variables, $\eta_{r}^{\text {det }}$ the detailed model prediction, and $\sigma_{r}^{\text {tol }}$ the error tolerance between the detailed and reduced model predictions. For the present analysis, $\sigma_{r}^{\text {tol }}$ is taken to be $2 \mathrm{~cm} / \mathrm{s}$ for laminar flame speeds and 0.2 in the natural logarithm for ignition delay times (equivalent to a factor of 1.22), which are in both cases comparable to the experimental uncertainty. The choices of these values are consistent with what have been discussed in a prior work [20].

Equation 4 can be approximated by a multivariate normal distribution, which will then have an $\mathbf{x}^{(0)^{*}}$ that best reproduces the detailed model, and a $\Sigma^{*}$ that best reproduces their uncertainty. $\quad \mathbf{x}^{(0)^{*}}$ is found by the mode of the PDF in Eq. 4, equivalent to the least-squares optimization problem

$$
\mathbf{x}^{(0)^{*}}=\underset{\mathbf{x}}{\arg \max } \ln P_{\mathrm{x}}(\mathbf{x})
$$

and solved using the LMDIF solver [64]. $\quad \Sigma^{*}$ is identified by linearizing the model predictions in the vicinity of $\mathbf{x}^{(0)^{*}}$, which yields

$$
\Sigma^{*}=\left[\sum_{i=1}^{M} \frac{\mathbf{J}_{r} \mathbf{J}_{r}^{T}}{\left(\sigma_{r}^{\mathrm{tol}}\right)^{2}}+4 \mathbf{I}\right]^{-1}
$$

where $\mathbf{J}_{r}$ is the gradient of $\eta_{r}\left(\mathbf{x}^{(0)^{*}}\right) \cdot \mathbf{x}^{(1)^{*}}$ is then determined through the Cholesky factorization of $\Sigma^{*}$. 
To reduce the computational complexity of Eqs. 5 and 6, the method of solution mapping [1, 65] was used, in which the reduced-model predictions are expressed as polynomials with respect to the reaction rate parameters, $\eta_{r}(\mathbf{X})=\mathbf{X}^{T} \mathbf{b}_{r} \mathbf{X}+\mathbf{a}_{r}^{T} \mathbf{X}+\eta_{r, 0}$, where $\mathbf{a}_{r}$ and $\mathbf{b}_{\mathrm{r}}$ are the first and second derivatives of $\eta_{r}$, and $\eta_{r, 0}$ is the nominal prediction of the model. The derivatives are calculated using a method based on sensitivity analysis [66]. For a given value of $\mathbf{x}^{(0)}$ and $\mathbf{x}^{(1)}$, the predictions $\eta_{r}$ have the PCE, $\eta_{r}(\xi)=\xi^{T} \hat{\boldsymbol{\beta}}_{r} \xi+\hat{\boldsymbol{\alpha}}_{r}^{T} \xi+\eta_{r}\left(\mathbf{x}^{(0)}\right)$, with $\hat{\boldsymbol{\beta}}_{r}=\mathbf{x}^{(1) T} \mathbf{b}_{r} \mathbf{x}^{(1)}$, and $\hat{\boldsymbol{\alpha}}_{r}=\mathbf{x}^{(1) T} \mathbf{J}_{r}$. The uncertainty $\sigma_{r}$ is calculated by taking the variance,

$$
\sigma_{r}^{2}=\mathrm{E}\left(\eta_{r}^{2}\right)-\mathrm{E}^{2}\left(\eta_{r}\right)=\sum_{i=1}^{N_{r}} \hat{\alpha}_{r, i}^{2}+2 \sum_{i=1}^{N_{r}} \hat{\beta}_{r, i i}^{2}+\sum_{i=1}^{N_{r}-1} \sum_{j=i+1}^{N_{r}} \hat{\beta}_{r, i j}^{2}
$$

To calculate the uncertainty in the reduced models before optimization, $\mathbf{x}^{(0)}=\mathbf{0}$ and $\mathbf{x}^{(1)}=\mathbf{I}_{N_{r}}$, i.e., the $N_{r}$-dimensional identity matrix, and so $\hat{\boldsymbol{\beta}}_{r}=\mathbf{b}_{r}, \hat{\boldsymbol{\alpha}}_{r}=\mathbf{a}_{r}$. The prediction uncertainty is given by $\sigma_{r}^{2}=\sum_{i=1}^{N_{r}} a_{r, i}^{2}+2 \sum_{i=1}^{N_{r}} b_{r, i i}^{2}+\sum_{i=1}^{N_{r}-1} \sum_{j=i+1}^{N_{r}} b_{r, i j}^{2}$.

\section{Results and Discussion}

\subsection{Skeletal Model Generation and Analysis}

The skeletal reduction was first conducted through the use of DRG, as discussed before. From detailed simulations of each auto-ignition and laminar premixed flame, the reaction states were sampled at typical temperatures, which start from the initial temperature of auto-ignition and the inlet temperature of the laminar premixed flame till the final state of simulation by the interval of $50 \mathrm{~K}$. The DRG index was calculated for each species at every sampled reaction state, and each species was assigned by its maximum DRG index among all cases to avoid the importance of species being under-represented. It was determined that the results do not depend on the sampling interval because the same skeletal models were generated by sampling in $25 \mathrm{~K}$ intervals. Figure 1 presents the correlation between the number of species, $N_{s}$, in the resulting 
skeletal model and the threshold value $\varepsilon$. In previous DRG studies [36-38], a threshold value of 0.1 was shown to be a reasonable cutoff. In the current work, however, an $\varepsilon$ value of 0.1 can only remove around ten species, which indicates the compact nature of USC Mech II. The model includes very few nonessential species. Because the benefit of applying DRG is limited in this case, this work eliminated no species by DRG.

In the DRGASA process that followed, ignition delay times $\tau$ and laminar flame speeds $s_{u}^{\circ}$ predicted by using USC Mech II were taken as the reference, training values. Spanning all thermodynamic conditions considered in this work, DRGASA calculates the maximum percentage error, $e r r_{\max }$, in computing these global responses caused by the removal of each species. Accordingly, a ranking list of species is decided by their impact on model predictions. Figure 2 correlates the error of model prediction to the model size. Two sets of three skeletal models are generated by truncating the model with respect to the error about $25 \%, 60 \%$ and $100 \%$ for the ignition delay prediction, one set each for $n$-butane and $i$-butane. The properties of skeletal models are shown in Table 2 . The $n-1$ and $i-1$ models are quite close to the detailed model in terms of their predictions for the conditions tested. In contrast, the inferior performance of the more reduced versions may be attributed to over-truncation and thus missing critical reaction pathways under certain circumstances. The computational time of a chemical model typically scales with the square of species number [27], and the reduced models developed in this work roughly require $8 \%$ to $20 \%$ computational time with respect to the full model.

A substantial fraction of the reactions and species identified as critical to flame propagation and induction zone of the ignition delay overlap. Take $n$-butane as an example. Figure 3 presents the number of species, $N_{s, \mathrm{c}}$, which are common to the skeletal models obtained individually from ignition and flame targets. Independent of the model size, around $90 \%$ of the species are found to be critical to both combustion properties. For a model of 50 species, the species that are only exist in the model derived from the auto-ignition are $\mathrm{CH}_{3} \mathrm{CCH}_{2}, i \mathrm{C}_{3} \mathrm{H}_{7}, \quad n \mathrm{C}_{4} \mathrm{H}_{5}, i \mathrm{C}_{4} \mathrm{H}_{7}$, 
$\mathrm{CH}_{2} \mathrm{CHCHCHO}$ and those that only exist in the model derived from the flames are $\mathrm{CH}, \mathrm{CH}_{2} \mathrm{OH}$, $\mathrm{C}_{2} \mathrm{H}, \mathrm{H}_{2} \mathrm{CC}, \mathrm{CH}_{3} \mathrm{CO}$. The intersection of the ranking lists emphasizes the importance of the small molecule chemistry in both cases; and the species unique to the auto-ignition process correspond to a particular set of $\mathrm{C}_{3}-\mathrm{C}_{4}$ fragments of importance to the initial fuel breakdown. Comparing the skeletal models of $n$-butane with $i$-butane derived from the auto-ignition simulations, around $77 \%$ of the species retained are identical, as shown in Figure 4. For a 50-species model, the species unique to $n$-butane are $n \mathrm{C}_{3} \mathrm{H}_{7}, \quad \mathrm{C}_{3} \mathrm{H}_{8}, \quad n \mathrm{C}_{4} \mathrm{H}_{5}, \quad i \mathrm{C}_{4} \mathrm{H}_{5}, \quad \mathrm{C}_{4} \mathrm{H}_{8}-2, \quad p \mathrm{C}_{4} \mathrm{H}_{9}, \quad s \mathrm{C}_{4} \mathrm{H}_{9}$, $\mathrm{CH}_{2} \mathrm{CHCHCHO}$, and $n-\mathrm{C}_{4} \mathrm{H}_{10}$ and those unique to $i$-butane are $\mathrm{CH}_{2} \mathrm{OH}, \mathrm{CH}_{3} \mathrm{CO}, p \mathrm{C}_{3} \mathrm{H}_{4}$, $\mathrm{CH}_{2} \mathrm{CHCO}, \mathrm{CH}_{3} \mathrm{COCH}_{3}, i \mathrm{C}_{4} \mathrm{H}_{9}, t \mathrm{C}_{4} \mathrm{H}_{9}, \mathrm{C}_{6} \mathrm{H}_{5}$, and $i-\mathrm{C}_{4} \mathrm{H}_{10}$. The two models share the same chemistry of small hydrocarbons $\mathrm{C}_{1}-\mathrm{C}_{2}, \mathrm{CO}$ and $\mathrm{H}_{2}$, and differ in reaction pathways that are specific to a given fuel, especially for the ignition phenomenon. Of course, a comparison of the fuel-specific, intermediate species yields useful conclusion about pertinent reaction pathways of fuel oxidation and perhaps even the molecular growth process, such analysis is well beyond the scope of the present investigation.

\subsection{Skeletal Model Optimization and Constraint}

Skeletal models $n-1$ and $i-1$ are constrained against the detailed model predictions of the combustion targets shown in Table 2. The predictions of the unconstrained and constrained skeletal models are compared to those of the full model, as shown in Figure 5. Clearly, the performances of the skeletal models are improved by the constraining process, which reduced the maximum error from around $25 \%$ to $6 \%$ for the ignition delay and 2 to $1 \%$ for the flame speed, as shown in Table 2 . The optimized $n-1$ and $i$-1 models are provided in the supplemental material. For $n$-butane and $i$-butane, the rate coefficients of 103 and 97 reactions are impacted by the

constraining process, respectively. Figures 6 shows that most of the impacted $A$ factors are within $20 \%$ of the original value, well within their range of uncertainty. Optimization of other skeletal models listed in Table 2 was not attempted; as was discussed in Section 2, these models 
were aggressively reduced and therefore can have missing reaction pathways, leading to a change in the underlying physics and thus reduced general predictability.

\subsection{Size-Dependent Model Uncertainty}

We take this opportunity to explore a related question concerning model uncertainty: the correlation between model size and uncertainty. Intuitively, the model uncertainty defined within the framework of MUM-PCE should increase with an increase in the model size, namely the number of model parameters. That is, the individual uncertainties of these parameters have a cumulative effect on the overall model uncertainty. This intuitive understanding is, in fact, only conditionally correct. The uncertainties of the sample skeletal models listed in Table 2 in predicting the ignition delay are shown in Figure 7. The uncertainties of models $n-1$ (without optimization) and $n-2$ are generally similar, and the same observation also holds for the $i-1$ (without optimization) and $i-2$ models. Yet for the skeletal model $n-3$ and $i-3$ with the smaller size, the model uncertainties can be both larger and smaller than those of the larger models, depending on the specific simulation scenarios. Similar observations can be made for the prediction of laminar flame speeds as well, as shown in Figure 8.

The non-monotonous size-dependent model uncertainty may be understood by considering an artificial model of $N-1$ parameters. This model gives some prediction $\eta_{r, 0}^{\prime}$, with first and second derivative vectors $\mathbf{a}_{r}^{\prime}$ and $\mathbf{b}_{r}^{\prime}$. The uncertainty $\sigma_{r}^{\prime}$, before optimization, in this prediction is given by

$$
\sigma_{r}^{\prime 2}=\sum_{i=1}^{N-1} a_{r, i}^{\prime 2}+2 \sum_{i=1}^{N-1} b_{r, i i}^{\prime 2}+\sum_{i=1}^{N-2} \sum_{j=i+1}^{N-1} b_{r, i j}^{\prime 2}
$$

If the model is enlarged by adding an $N^{\text {th }}$ parameter, it would now have a prediction $\eta_{r, 0}$ and updated first and second derivative vectors $\mathbf{a}_{r}$ and $\mathbf{b}_{r}$. The updated model uncertainty $\sigma_{r}$ will be 


$$
\sigma_{r}^{2}=\sum_{i=1}^{N} a_{r, i}^{2}+2 \sum_{i=1}^{N} b_{r, i i}^{2}+\sum_{i=1}^{N-1} \sum_{j=i+1}^{N} b_{r, i j}^{2}
$$

If the $N^{\text {th }}$ parameter does not influence the prediction and the sensitivity coefficients of the first through the $(N-1)^{\text {th }}$ parameters, $\mathbf{a}_{r}$ and $\mathbf{b}_{r}$ share the corresponding components with $\mathbf{a}_{r}^{\prime}$ and $\mathbf{b}_{r}^{\prime}$. The variance $\sigma_{r}^{2}$ can be split into

$$
\sigma_{r}^{2}=\sigma_{r}^{\prime 2}+a_{r, N}^{2}+2 b_{r, N N}^{2}+\sum_{i=1}^{N-1} b_{r, i N}^{2}
$$

which implies the model uncertainty must increase or at least remain constant with the model size, because of the non-negative term $a_{N, N}^{2}+2 b_{N, N N}^{2}+\sum_{i=1}^{N-1} b_{N, i N}^{2}$. If the $N^{\text {th }}$ parameter is weakly coupled to the existing $N-1$ parameters, the model uncertainty will increase or at least remain constant. In contrast, if the $N^{\text {th }}$ parameter impacts the sensitivities of the first through $(N-1)^{\text {th }}$ parameters with respect to the model prediction, the values of $\mathbf{a}_{r}$ and $\mathbf{b}_{r}$ must be identified anew and thereafter the behavior of $\sigma_{r}$ and $\sigma_{r}^{\prime}$ cannot be predicted $a$ priori. In other words, the dependence of reaction model uncertainty on the model size is subject to the extent of coupling within model parameters. For the case of strong coupling, the sensitivity of a particular combustion response to the rate parameters may be substantially affected by pathway truncation. Such a condition is indicative of the truncation of critical reaction pathways during model reduction. Hence, the uncertainty of a reduced model, when compared to that of a full model, is indicative of the truncation of the necessary physics in model reduction, as expected.

To verify the above argument in real combustion system, we conducted a prototype study on the auto-ignition of stoichiometric $n$-butane-air mixture under atmospheric pressure, with an initial temperature of $1000 \mathrm{~K}$, where the model uncertainty non-monotonically changes, first decreasing with the model size and increases afterwards. The $n-1$ model (without optimization) contains all species and reactions contained within the $n-2$ model, which in turn contains the species and reactions within the $n-3$ model. The set of extra reactions contained in $n-1$ but not in 
$n-2$ and those contained in $n-2$ but not in $n-3$ are termed as $\mathrm{R}_{1-2}$ and $\mathrm{R}_{2-3}$, respectively. The coupling extent of the $i^{\text {th }}$ reaction with other reactions is investigated by comparing the magnitude of the $a_{i}$ and $b_{i j}$ terms. A strong coupling reaction will be represented by a large $b_{i j}$ term. For reactions within sets $\mathrm{R}_{2-3}$ and $\mathrm{R}_{1-2}$, the magnitudes of the corresponding $a_{i}$ and $b_{i j}$ are illustrated in Fig. 9. The first order coefficients are presented as $\left|a_{i}\right|$ and the second order coefficients by the average value, $\left|\bar{b}_{i}\right|=1 / N_{r} \sum_{j=1}^{N_{r}}\left|b_{i j}\right|$. In the reaction set $\mathrm{R}_{2-3}$, the $a_{i}$ and $b_{i j}$ values are comparable, indicating strong coupling between the reactions. This explains the large change in the prediction uncertainty between the models $n-2$ and $n-3$. In the reaction set $\mathrm{R}_{1-2}$, the $a_{i}$ values are much larger than the $b_{i j}$ values, and there is correspondingly a small increase in the prediction uncertainties from models $n-2$ to $n-1$. That is, the model uncertainty increases, but only slightly, as the model size is increased.

The above observation leads to a question not previously addressed but it requires some consideration here. That is, whether the model prediction uncertainty is convergent with respect to model size. We note that a fully complete reaction model must consider all binary interactions. For USC Mech II with 108 reactive species, a full, combinatory description must consider $>10,000$ reactions if all of them have only one channel. Of course, each reaction can have and is often associated with a very large number of pathways (and those considered in a conventional reaction models are only the lower-lying potential energy paths). In that regard, all detailed reaction models have already been truncated or reduced to an extent at the onset of model building. Theoretically, additional terms added to Eq. (9) due to an increasing number of reactions $N$ should lead to a convergent sum. This can be proved mathematically by comparing the individual terms of the series of Eq. (9) with the terms of a convergent series. Figure 10 compares the decay rates of $\mathbf{a}_{\mathrm{r}}$ and $\mathbf{b}_{r}$ with the reciprocals of positive integer numbers $\left(i^{-1}\right.$, $i=, 1,2, . . \infty)$. Note that the series sum of the reciprocals of square numbers $\sum_{i=1}^{\infty} i^{-2}$ is convergent. The prediction uncertainty, from Eq. (9), is the sum of the squares of $\mathbf{a}_{\mathrm{r}}$ and $\mathbf{b}_{r}$, and so the fact 
that $\mathbf{a}_{\mathrm{r}}$ and $\mathbf{b}_{r}$ terms decay faster than $i^{-1}$ indicates that Eq. (9) is convergent for the $n$-butane oxidation problem considered. Of course, we expect the comparison to hold also for other combustion problems.

\section{Conclusions}

Reduced chemistry models for $n$-butane and $i$-butane combustion are derived from USC Mech II by skeletal reduction and model optimization. The yielded models reproduce the prediction of the detailed model. The rate coefficients of the skeletal model are adjusted systematically within their uncertainty bounds using MUM-PCE.

The relationship between the model size and the uncertainty of its predictions is explored using three sample skeletal models of different sizes. The completeness, and therefore the reliability, of a reduced model can be assessed by the behavior of the uncertainty of its predictions with respect to the model size. This behavior can also be assessed by the second derivatives of the model predictions with respect to the rate parameters, or in other words the inter-parameter coupling. As long as reactions being removed from the reduced model do not exhibit this second-order coupling with reactions that remain, the model prediction uncertainty increases with an increase in the model size in principle, and this uncertainty should always be convergent as the model approaches completeness. For a model that has reactions removed that exhibit second-order coupling with the reactions retained the reduced model, the dependence of the prediction uncertainty on the model size may be unpredictable. 


\section{Acknowledgements}

Work at Princeton and Stanford was supported by the Combustion Energy Frontier Research Center (CEFRC), an Energy Frontier Research Center funded by the U.S. Department of Energy, Office of Science, Office of Basic Energy Sciences under Award Number DE-SC0001198.

\section{Disclaimer}

Certain trade names are identified in this paper in order to specify the computational procedure adequately. Such identification is not intended to imply recommendation or endorsement by the National Institute of Standards and Technology, nor is it intended to imply that the trade names identified are necessarily the best available for the purpose. 


\section{Reference}

1. M. Frenklach, H. Wang, M. J. Rabinowitz, Prog. Energ. Combust. Sci. 18 (1992) 47-73.

2. T. Turanyi, L. Zalotai, S. Dobe, T. Berces, Phys. Chem. Chem. Phys. 4 (2002) 2568-2578.

3. B. Bhattacharjee, D. A. Schwer, P. I. Barton, W. H. Green, Combust. Flame 135 (2003) 191-208.

4. M. T. Reagan, H. N. Najm, R. G. Ghanem, O. M. Knio, Combust. Flame 132 (2003) 545-555.

5. M. T. Reagan, H. N. Najm, B. J. Debusschere, O. P. Le Maitre, M. Knio, R. G. Ghanem, Combust. Theor. Model. 8 (2004) 607-632.

6. M. T. Reagan, H. N. Najm, P. P. Pebay, O. M. Knio, R. G. Ghanem, Int. J. Chem. Kinet. 37 (2005) 368-382.

7. J. Zador, I. G. Zsely, T. Turanyi, M. Ratto, S. Tarantola, A. Saltelli, J. Phys. Chem. A 109 (2005) 9795-9807.

8. I. G. Zsely, J. Zador, T. Turanyi, Proc. Combust. Inst. 30 (2005) 1273-1281.

9. $\quad$ P. Seiler, M. Frenklach, A. Packard, R. Feeley, Optim. Eng. 7 (2006) 459-478.

10. A. S. Tomlin, Reliab. Eng. Syst. Safe. 91 (2006) 1219-1231.

11. J. Zador, I. G. Zsely, T. Turanyi, Reliab. Eng. Syst. Safe. 91 (2006) 1232-1240.

12. M. Frenklach, Proc. Combust. Inst. 31 (2007) 125-140.

13. T. Russi, A. Packard, R. Feeley, M. Frenklach, J. Phys. Chem. A 112 (2008) 2579-2588.

14. D. A. Sheen, X. You, H. Wang, T. Løvås, Proc. Combust. Inst. 32 (2009) 535-542.

15. T. Russi, A. Packard, M. Frenklach, Chem. Phys. Lett. 499 (2010) 1-8.

16. M. J. Davis, R. T. Skodje, A. S. Tomlin, J. Phys. Chem. A 115 (2011) 1556-1578.

17. S. J. Klippenstein, L. B. Harding, M. J. Davis, A. S. Tomlin, R. T. Skodje, Proc. Combust. Inst. 33 (2011) 351-357. 
18. T. Nagy, T. Turanyi, Int. J. Chem. Kinet. 43 (2011) 359-378.

19. D. A. Sheen, H. Wang, Combust. Flame 158 (2011) 645-656.

20. D. A. Sheen, H. Wang, Combustion and Flame 158 (2011) 2358-2374.

21. L. Varga, B. Szabo, I. G. Zsely, A. Zempleni, T. Turanyi, J. Math. Chem. 49 (2011) 1798-1809.

22. X. Q. You, T. Russi, A. Packard, M. Frenklach, Proc. Combust. Inst. 33 (2011) 509-516.

23. T. Nagy, T. Turanyi, Reliab. Eng. Syst. Safe. 107 (2012) 29-34.

24. C. F. Goldsmith, A. S. Tomlin, S. J. Klippenstein, Proc. Combust. Inst. 34 (2013) 177-185.

25. D. A. Sheen, C. M. Rosado-Reyes, W. Tsang, Proc. Combust. Inst. 34 (2013) 527-536.

26. C. K. Westbrook, W. J. Pitz, O. Herbinet, H. J. Curran, E. J. Silke, Combust. Flame 156 (2009) 181-199.

27. T. Lu, C. K. Law, Prog. Energy Combust. Sci. 35 (2009) 192-215.

28. C. K. Law, C. J. Sung, H. Wang, T. Lu, AIAA J. 41 (2003) 1629-1646.

29. M. Frenklach, in: Numerical Approaches to Combustion Modeling, E. S. Oran; J. P. Boris, (Eds.) American Institute of Aeronautics and Astronautics, Inc.: Washington, DC, 1991; Vol. 135, pp 129-154.

30. A. S. Tomlin, T. Turányi, M. J. Pilling, in: Comprehensive Chemical Kinetics, M. J. Pilling, (Ed.) Elsevier: 1997; Vol. Volume 35, pp 293-437.

31. H. Rabitz, M. Kramer, D. Dacol, Ann. Rev. Phys. Chem. 34 (1983) 419-461.

32. T. Turányi, J. Math. Chem. 5 (1990) 203-248.

33. T. Turanyi, New journal of chemistry 14 (1990) 795-803.

34. S. Vajda, P. Valko, T. Turanyi, Int. J. Chem. Kinet. 17 (1985) 55-81.

35. H. Wang, M. Frenklach, Combust. Flame 87 (1991) 365-370.

36. T. Lu, C. K. Law, Proc. Combust. Inst. 30 (2005) 1333-1341.

37. T. Lu, C. K. Law, Combust. Flame 146 (2006) 472-483.

38. T. Lu, C. K. Law, Combust. Flame 144 (2006) 24-36. 
39. P. Pepiot-Desjardins, H. Pitsch, Combust. Flame 154 (2008) 67-81.

40. R. Sankaran, E. R. Hawkes, J. H. Chen, T. Lu, C. K. Law, Proc. Combust. Inst. 31 (2007) 1291-1298.

41. X. Zheng, T. Lu, C. Law, Proc. Combust. Inst. 31 (2007) 367-375.

42. $\quad$ N. Peters, F. Williams, Combust. Flame 68 (1987) 185-207.

43. J.-Y. Chen, Combust. Sci. Technol. 57 (1988) 89-94.

44. C. Sung, C. Law, J.-Y. Chen, Combust. Flame 125 (2001) 906-919.

45. T. Løvås, F. Mauss, C. Hasse, N. Peters, Proc. Combust. Inst. 29 (2002) 1403-1410.

46. H. S. Soyhan, F. Mauss, C. Sorusbay, Combust. Sci. Technol. 174 (2002) 73-91.

47. T. Mendiara, M. Alzueta, A. Millera, R. Bilbao, Energy Fuel. 18 (2004) 619-627.

48. A. Massias, D. Diamantis, E. Mastorakos, D. Goussis, Combust. Flame 117 (1999) 685-708.

49. A. Massias, D. Diamantis, E. Mastorakos, D. A. Goussis, Combust. Theor. Model. 3 (1999) 233-257.

50. T. Lu, Y. Ju, C. K. Law, Combust. Flame 126 (2001) 1445-1455.

51. C. J. Montgomery, C. Yang, A. R. Parkinson, J.-Y. Chen, Combust. Flame 144 (2006) $37-52$.

52. T. Lu, C. K. Law, Combust. Flame 154 (2008) 761-774.

53. U. Maas, S. B. Pope, Combust. Flame 88 (1992) 239-264.

54. S. Lam, D. Coussis in: Understanding complex chemical kinetics with computational singular perturbation, Symposium (International) on Combustion, 1989; Elsevier: 1989; pp 931-941.

55. S. Lam, D. Goussis, Int. J. Chem. Kinet. 26 (1994) 461-486.

56. M. Valorani, H. N. Najm, D. A. Goussis, Combust. Flame 134 (2003) 35-53.

57. M. Valorani, F. Creta, D. A. Goussis, J. C. Lee, H. N. Najm, Combust. Flame 146 (2006) 29-51. 
58. H. Wang, X. You, A. Joshi, S. Davis, A. Laskin, F. Egolfopoulos, C. Law, USC Mech Version II. High-Temperature Combustion Reaction Model of H2/CO/C1-C4 Compounds. http://ignis.usc.edu/Mechanisms/USC-Mech\%20II/USC_Mech\%20II.htm; 2007

59. P. N. Brown, G. D. Byrne, A. C. Hindmarsh, SIAM J. Sci. Stat. Comp. 10 (1989) 1038-1051.

60. R. J. Kee, F. M. Rupley, J. A. Miller, CHEMKIN-II: A FORTRAN Chemical Kinetics Package for the Analysis of Gas-Phase Chemical Kinetics, Sandia National Laboratories: Albequerque, NM, 1989.

61. R. Kee, J. Grcar, M. Smooke, J. Miller, E. Meeks, PREMIX: AFORTRAN Program for Modeling Steady Laminar One-Dimensional Premixed Flames, Sandia National Labs: Albequerque, NM, 1986.

62. R. J. Kee, G. Dixon-Lewis, J. Warnatz, M. E. Coltrin, J. A. Miller, A FORTRAN Computer Code Package for the Evaluation of Gas-Phase Viscosities, Conductivities, and Diffusion Coefficients, Sandia National Labs: Albequerque, NM., 1986.

63. P. Middha, H. Wang, Combust. Theor. Model. 9 (2005) 353-363.

64. J. More, B. Garbow, K. Hillstrom, User Guide for MINPACK-1 Technical Report ANL-80-74, Argonne National Laboratory, 1980.

65. M. Frenklach, Combust. Flame 58 (1984) 69-72.

66. S. G. Davis, A. B. Mhadeshwar, D. G. Vlachos, H. Wang, Int. J. Chem. Kinet. 36 (2004) 94-106. 
Table 1. Combustion targets used to reduce and constrain the model

\begin{tabular}{ccc}
\hline Target $^{a}$ & Flame speeds & Ignition delay times \\
\hline$p(\mathrm{~atm})$ & $1,10,20$ & $1,10,20$ \\
$\varphi$ & $0.6,1,1.5$ & $0.6,1,1.5$ \\
$T_{0} / T_{\text {in }}(\mathrm{K})$ & 300 & $800,1000,1200,1400,1500$ \\
\hline
\end{tabular}

${ }^{a}$ The targets are constituted by taking the combination of pressures, equivalence ratios and initial/inlet temperatures.

Table 2. Summary of skeletal models.

\begin{tabular}{rcccc}
\hline Model & $N_{\mathrm{S}}$ & $N_{R}$ & \multicolumn{2}{c}{$\boldsymbol{e r r _ { \operatorname { m a x } } ( \% ^ { a } )}{ }^{\circ}$} \\
\hline$n$-butane & & & & $s_{u}$ \\
$n-1$ & 49 & 384 & $27.6^{b} / 6.3^{c}$ & $2.3^{b} / 1.1^{c}$ \\
$n-2$ & 43 & 309 & 60.4 & 3.8 \\
$n-3$ & 31 & 152 & 100.0 & 11.1 \\
$i$-butane & & & & \\
$i-1$ & 54 & 402 & $24.7^{b} / 6.4^{c}$ & $2.4^{b} / 1.2^{c}$ \\
$i-2$ & 47 & 343 & 61.3 & 5.0 \\
$i-3$ & 38 & 233 & 100.0 & 12.6 \\
\hline
\end{tabular}

${ }^{a}$ The relative error reported is selected as the worst case.

${ }^{b}$ Before optimization.

${ }^{c}$ After optimization. 


\section{Figure Captions}

Figure 1. Dependence of number of species in a skeletal model on the threshold value $\varepsilon$ as determined by DRG based on auto-ignition delay and laminar flame speed of $n$-butane-air and $i$-butane-air mixtures over the range of thermodynamic and mixture conditions considered (see text).

Figure 2. Dependence of maximum percentage deviation from the full model on the model size, calculated for auto-ignition delay and laminar flame speed of $n$-butane-air and $i$-butane-air mixtures over the range of thermodynamic and mixture conditions considered (see text).

Figure 3. Number of common species versus the total number of species in the skeletal models, developed for auto-ignition delay and laminar flame speed of $n$-butane-air mixtures over the entire range of thermodynamic and mixture conditions considered. Symbols are computational data; the line represents a linear fit to the data.

Figure 4. Number of common species versus the total number of species in the skeletal models developed for auto-ignition delay of $n$-butane-air and $i$-butane-air mixtures over the entire range of thermodynamic and mixture conditions considered. Symbols are computational data; the line represents a linear fit to the data.

Figure 5. $45^{\circ}$ degree diagonal plot of ignition delays predicted by the skeletal models versus the detailed model (left panel) and laminar flame speeds as a function of the equivalence ratio (right panel) for $n$-butane-air and $i$-butane-air mixtures using models $n$ - 1 and $i$-1, respectively. Star and circle represent the predictions made by the skeletal models with and without optimization; the line represents the prediction made by the detailed model.

Figure 6. Changes in the $A$ factors in the constrained skeletal models $n$-1 (top) and $i$-1 (bottom). $i$ represents the reaction index.

Figure 7. Uncertainties of three skeletal models for $n$-butane-air (a) and $i$-butane-air (b) mixtures in predicting ignition delays. Symbols represent the standard deviation of model prediction, $\sigma$, calculated for auto-ignitions at pressures of 1, 10 and $20 \mathrm{~atm}$, initial temperature from 1000 to $1400 \mathrm{~K}$ and equivalence ratio from 0.6 to 1.5 ; lines are drawn to guide the eye.

Figure 8. Uncertainties of various skeletal models for $n$-butane-air (left panel) and $i$-butane-air (right panel) mixtures in predicting flame speeds. Symbols represent the standard deviation of model prediction, $\sigma$, calculated for premixed flames at pressures 1, 10 and $20 \mathrm{~atm}$, inlet temperature of $300 \mathrm{~K}$ and equivalence ratio from 0.6 to 1.5 ; lines are drawn to guide the eye. 
Figure 9. Magnitude of derivative of reaction sets $\mathrm{R}_{2-3}$ and $\mathrm{R}_{1-2}$ computed for the auto-ignition of $n$-butane-air mixture under pressure $1 \mathrm{~atm}$, initial temperature of $1000 \mathrm{~K}$ and unity equivalence ratio. $i$ is the reaction index and symbols represent the absolute values of the first and second-order derivatives. Lines are drawn to guide the eye.

Figure 10. Decay rate of $\mathbf{a}_{\mathrm{r}}$ and $\mathbf{b}_{r}$ of eq. (9) (solid lines) calculated for the auto-ignition of $n$-butane at the pressure $1 \mathrm{~atm}$, initial temperature $1000 \mathrm{~K}$ and unity equivalence ratio. The $\mathbf{b}_{r}$ values are presented as the average $\left|\bar{b}_{i}\right|=1 / N_{r} \sum_{j=1}^{N_{r}}\left|b_{i j}\right| i$ is the reaction index. Dashed-dotted-dashed lines represent the series of reciprocals of positive integer numbers. 


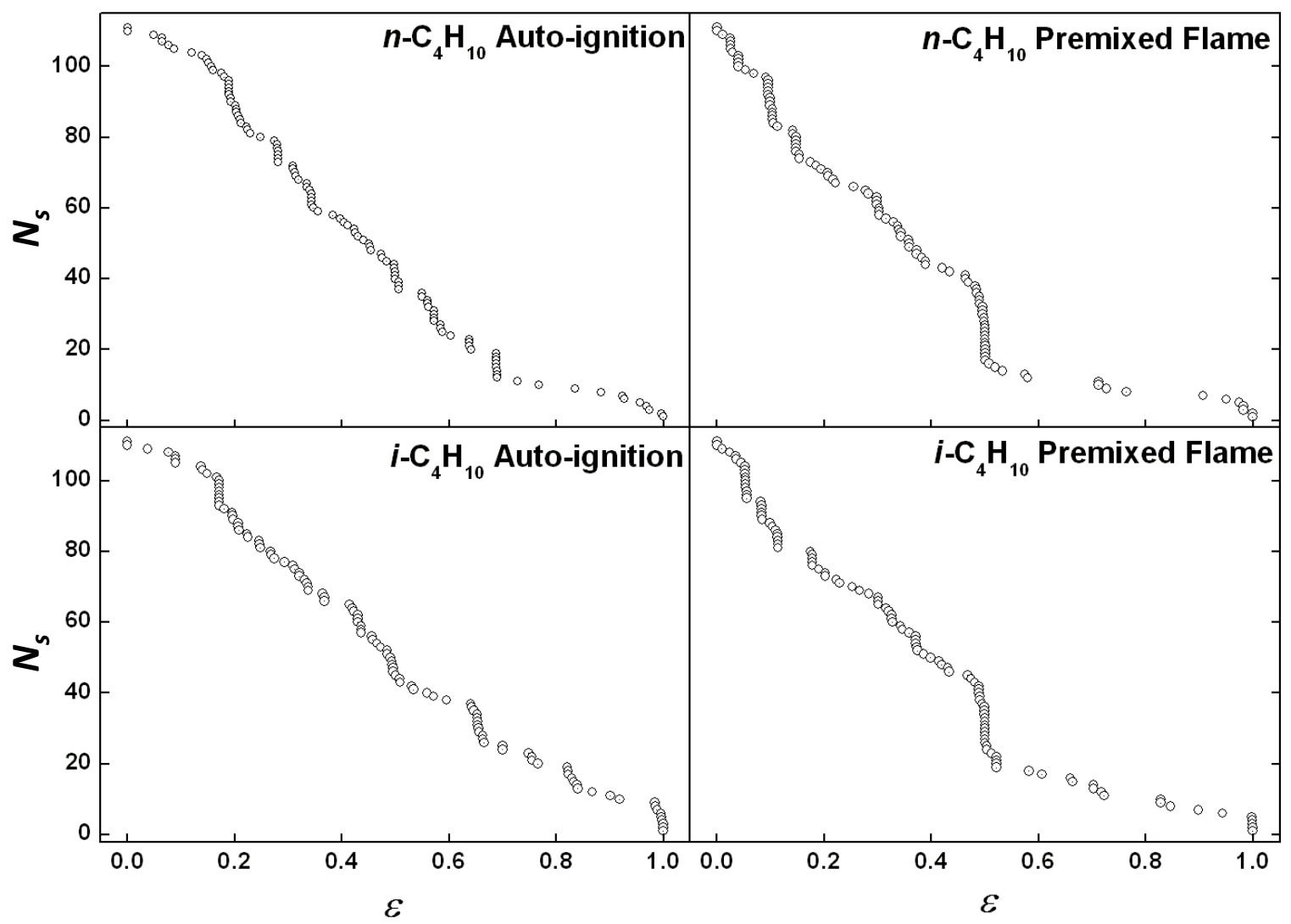

Figure 1. Dependence of number of species in a skeletal model on the threshold value $\varepsilon$ as determined by DRG based on auto-ignition delay and laminar flame speed of $n$-butane-air and $i$-butane-air mixtures over the range of thermodynamic and mixture conditions considered (see text). 


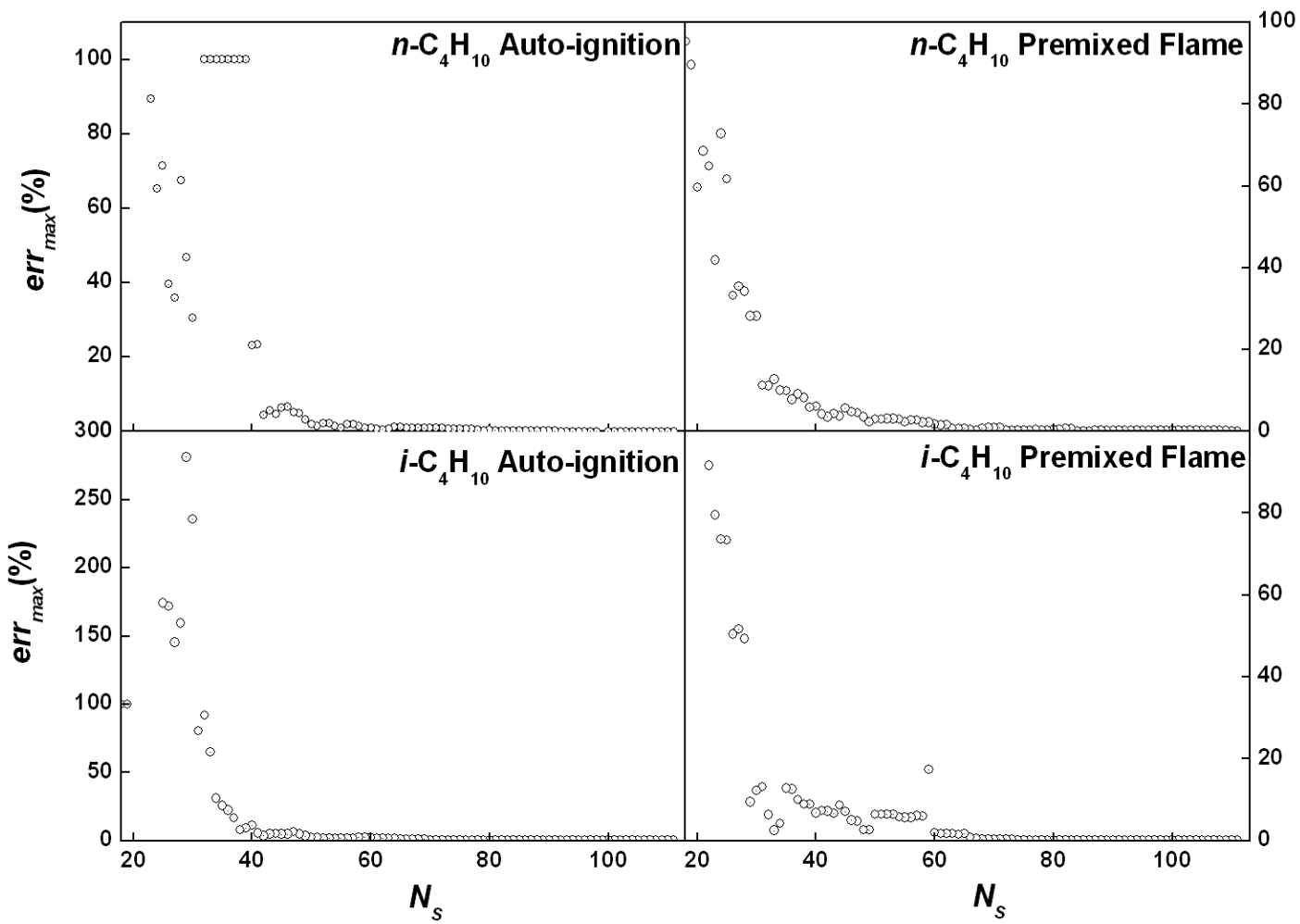

Figure 2. Dependence of maximum percentage deviation from the full model on the model size, calculated for auto-ignition delay and laminar flame speed of $n$-butane-air and $i$-butane-air mixtures over the range of thermodynamic and mixture conditions considered (see text). 


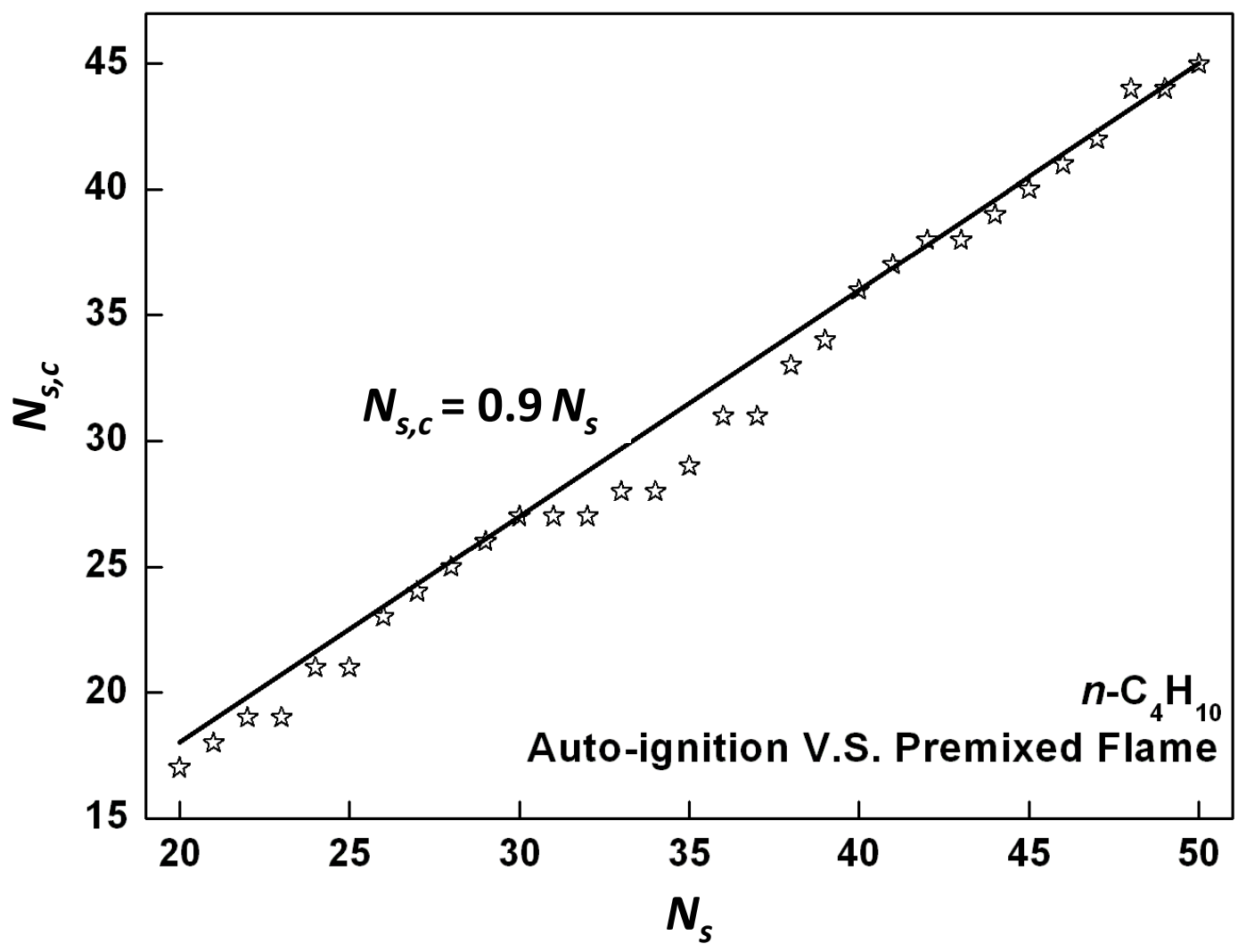

Figure 3. Number of common species versus the total number of species in the skeletal models, developed for auto-ignition delay and laminar flame speed of $n$-butane-air mixtures over the entire range of thermodynamic and mixture conditions considered. Symbols are computational data; the line represents a linear fit to the data. 


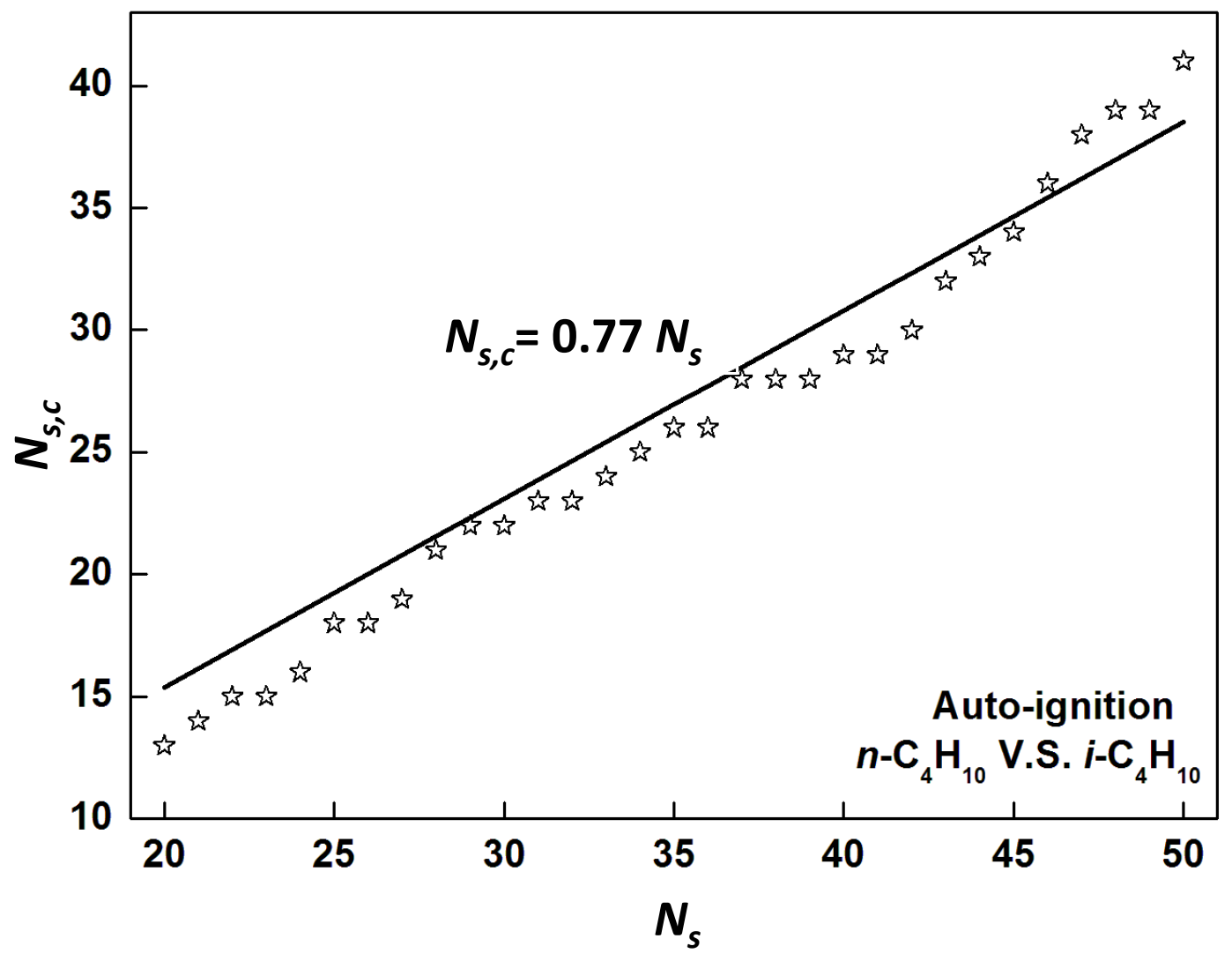

Figure 4. Number of common species versus the total number of species in the skeletal models developed for auto-ignition delay of $n$-butane-air and $i$-butane-air mixtures over the entire range of thermodynamic and mixture conditions considered. Symbols are computational data; the line represents a linear fit to the data. 


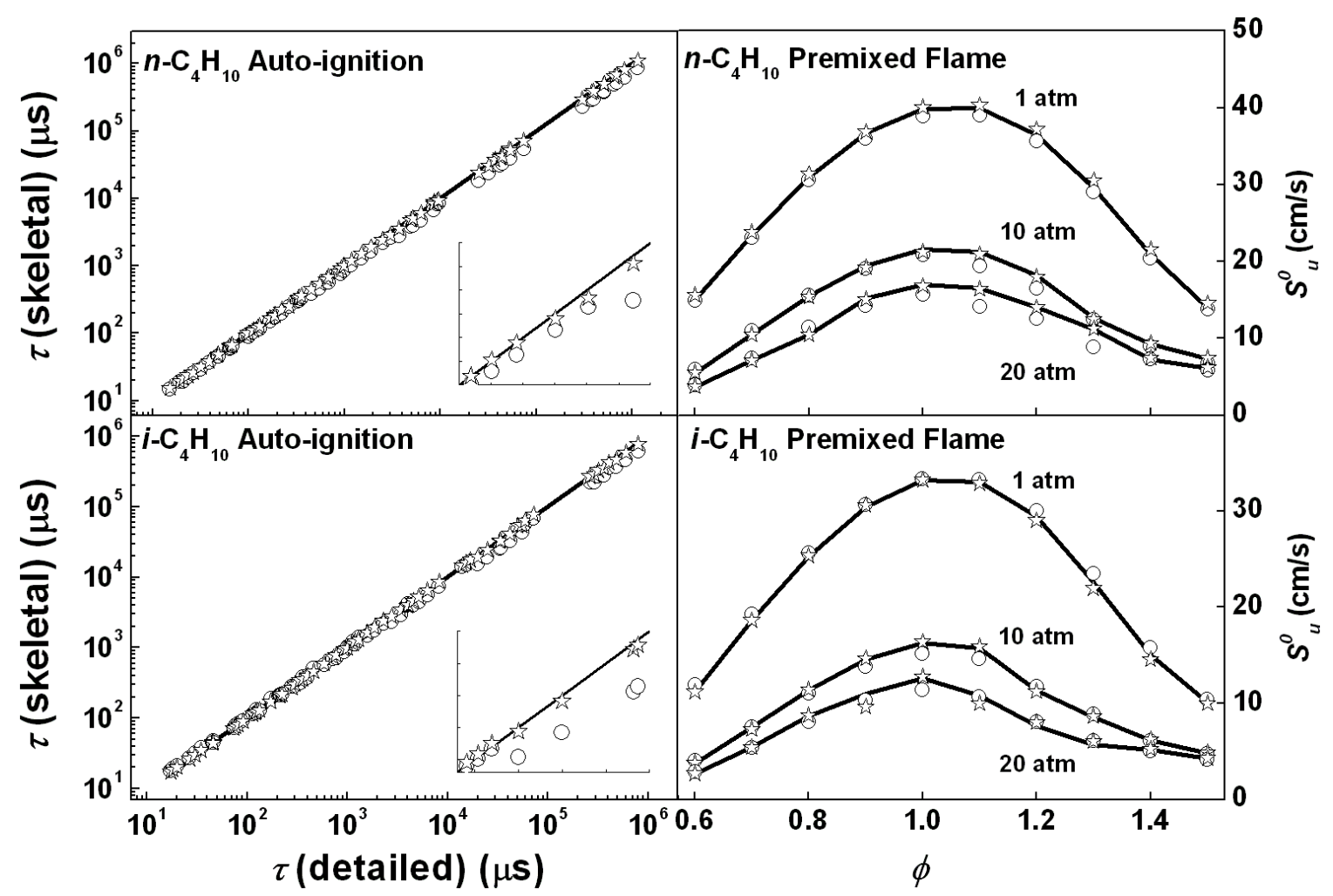

Figure 5. $45^{\circ}$ degree diagonal plot of ignition delays predicted by the skeletal models versus the detailed model (left panel) and laminar flame speeds as a function of the equivalence ratio (right panel) for $n$-butane-air and $i$-butane-air mixtures using models $n$ - 1 and $i$-1, respectively. Star and circle represent the predictions made by the skeletal models with and without optimization; the line represents the prediction made by the detailed model. 


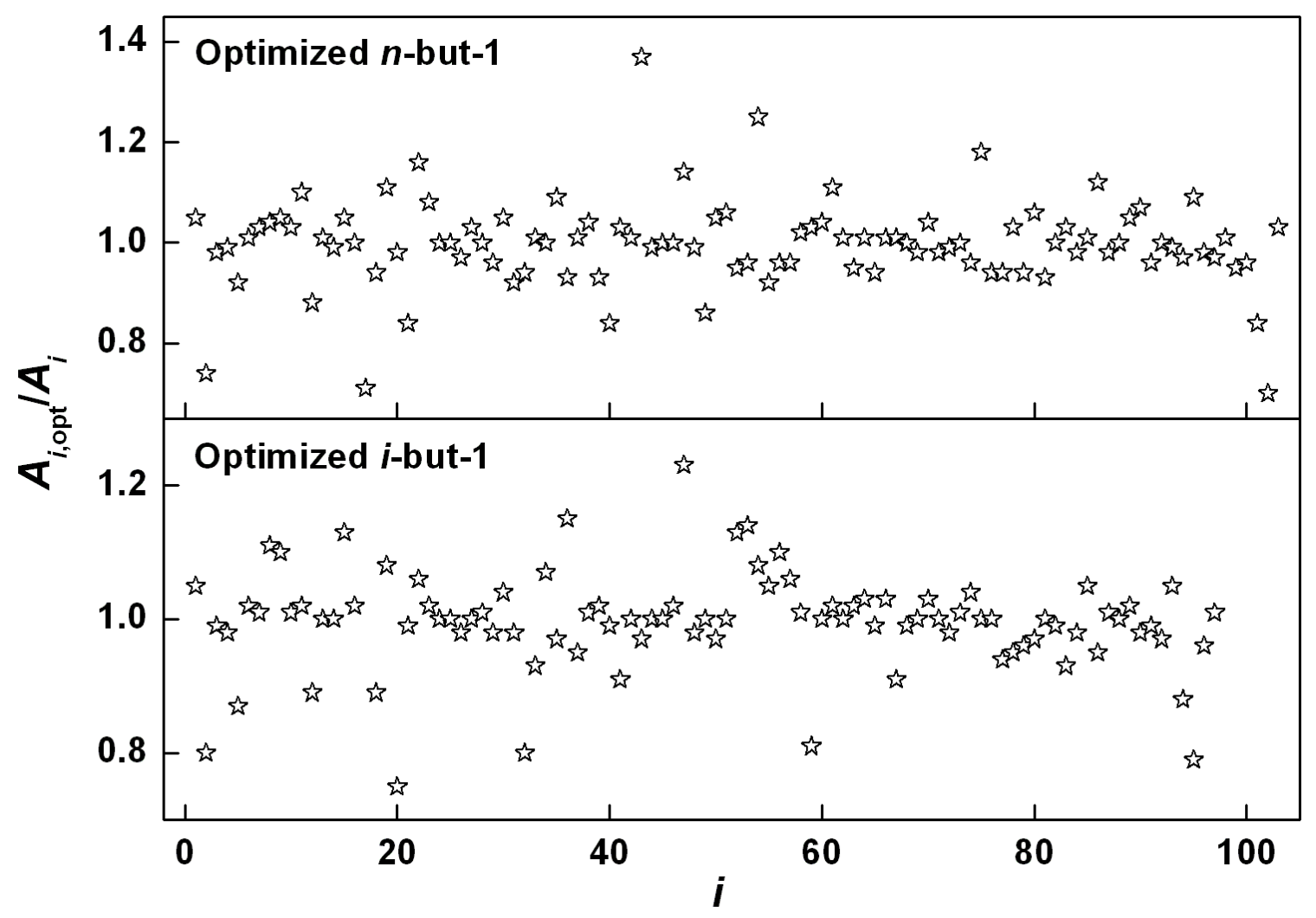

Figure 6. Changes in the $A$ factors in the constrained skeletal models $n-1$ (top) and $i-1$ (bottom). $i$ represents the reaction index. 

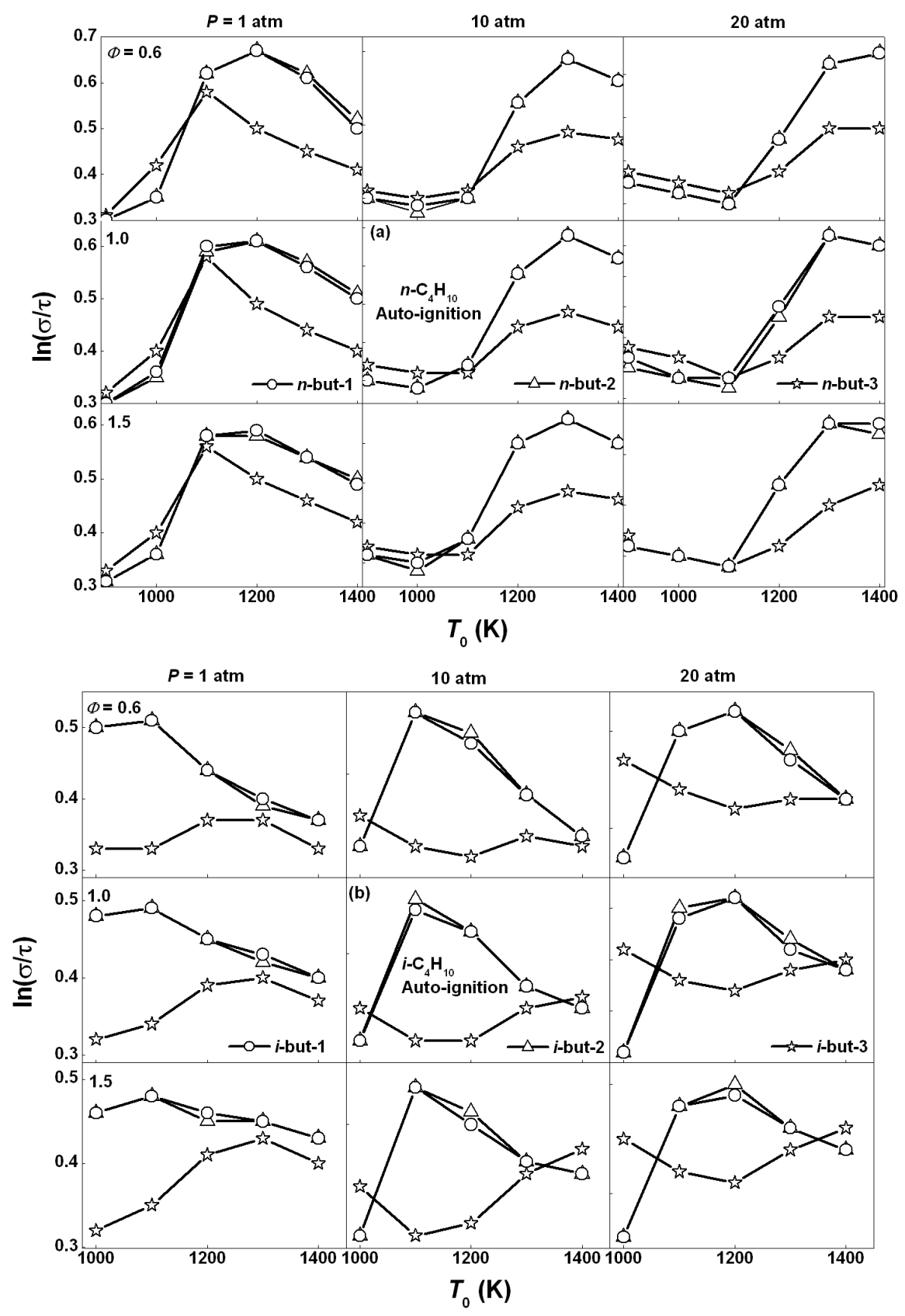

Figure 7. Uncertainties of three skeletal models for $n$-butane-air (a) and $i$-butane-air (b) mixtures in predicting ignition delays. Symbols represent the standard deviation of model prediction, $\sigma$, calculated for auto-ignitions at pressures of 1, 10 and $20 \mathrm{~atm}$, initial temperature from 1000 to $1400 \mathrm{~K}$ and equivalence ratio from 0.6 to 1.5 ; lines are drawn to guide the eye. 


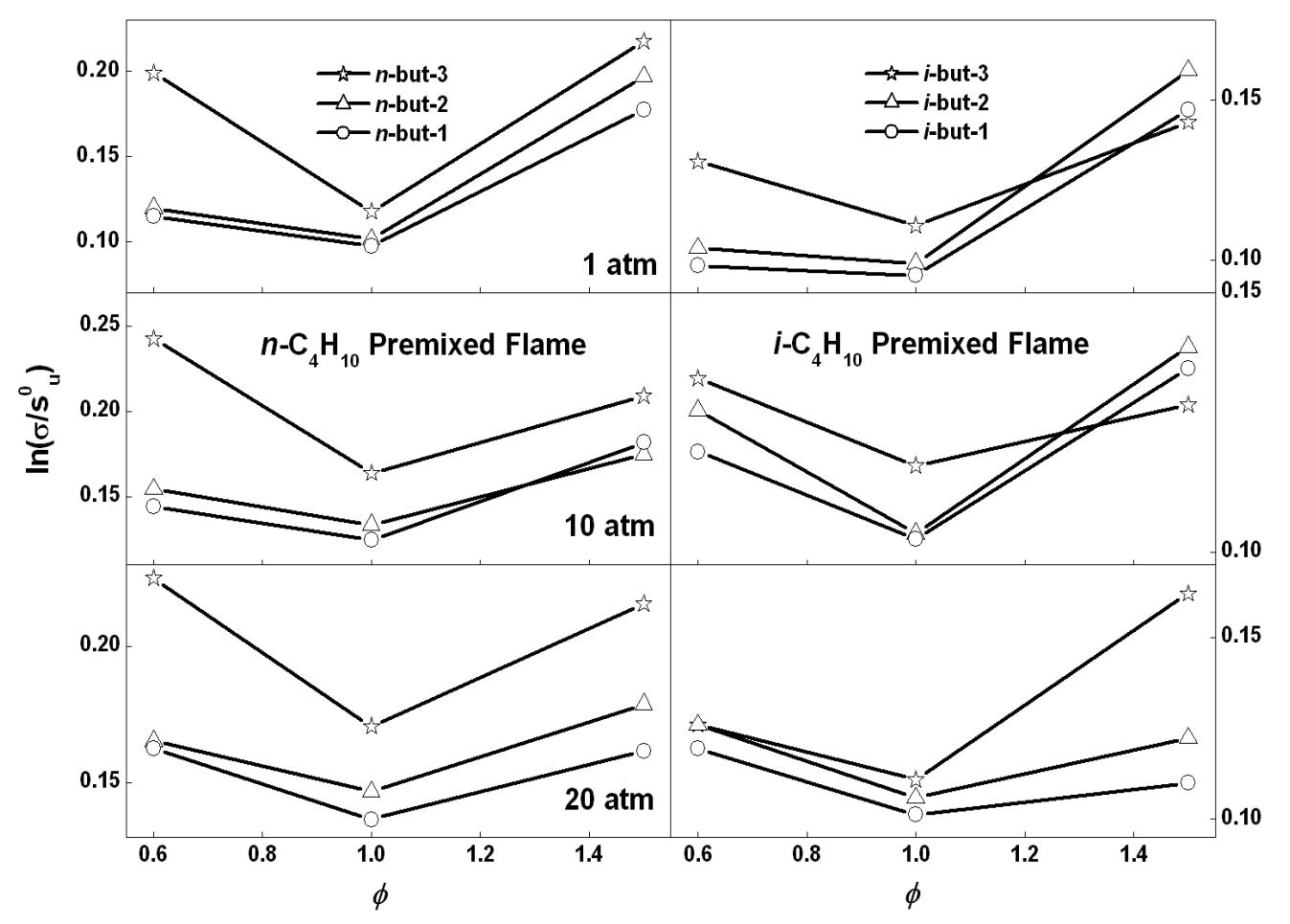

Figure 8. Uncertainties of various skeletal models for $n$-butane-air (left panel) and $i$-butane-air (right panel) mixtures in predicting flame speeds. Symbols represent the standard deviation of model prediction, $\sigma$, calculated for premixed flames at pressures 1,10 and 20 atm, inlet temperature of $300 \mathrm{~K}$ and equivalence ratio from 0.6 to 1.5 ; lines are drawn to guide the eye. 


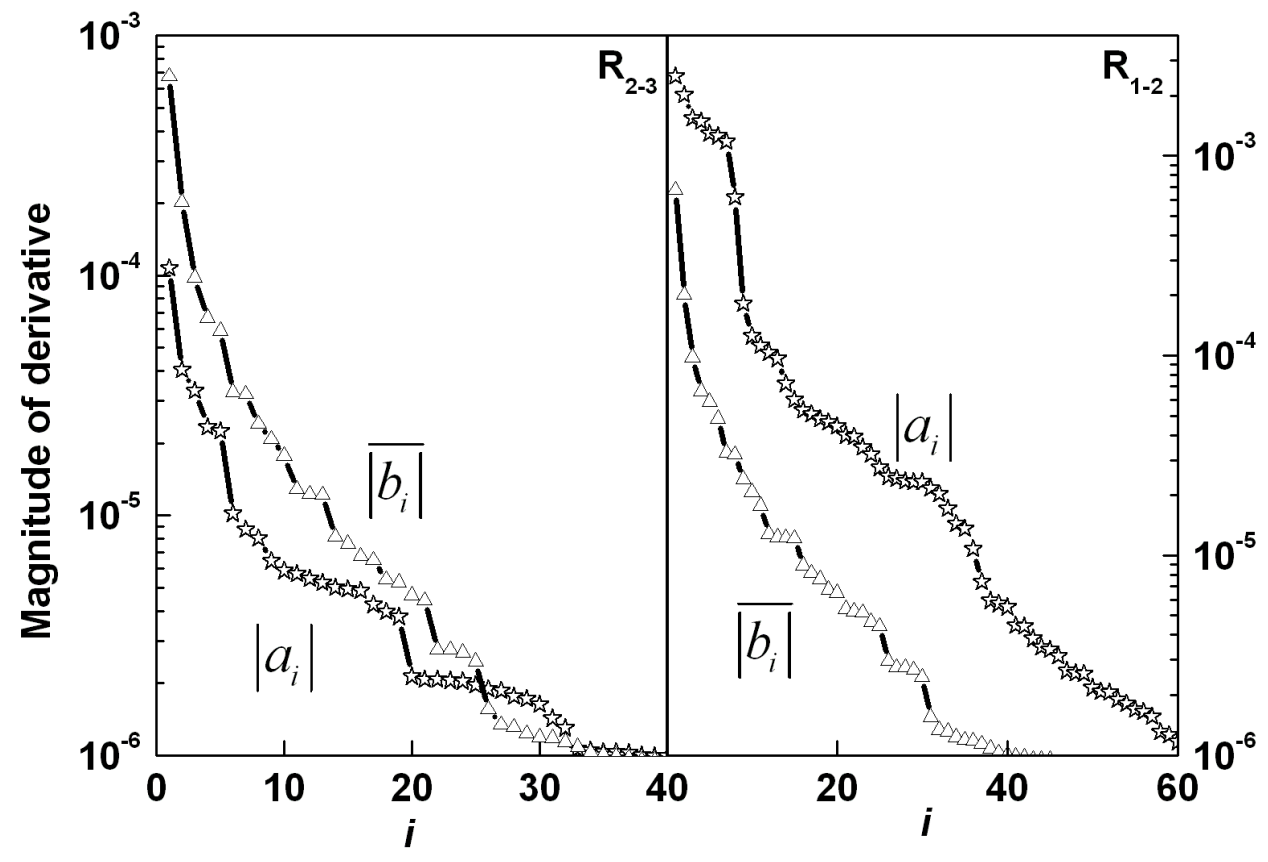

Figure 9. Magnitude of derivative of reaction sets $\mathrm{R}_{2-3}$ and $\mathrm{R}_{1-2}$ computed for the auto-ignition of $n$-butane-air mixture under pressure 1 atm, initial temperature of $1000 \mathrm{~K}$ and unity equivalence ratio. $i$ is the reaction index and symbols represent the absolute values of the first and second-order derivatives. Lines are drawn to guide the eye. 


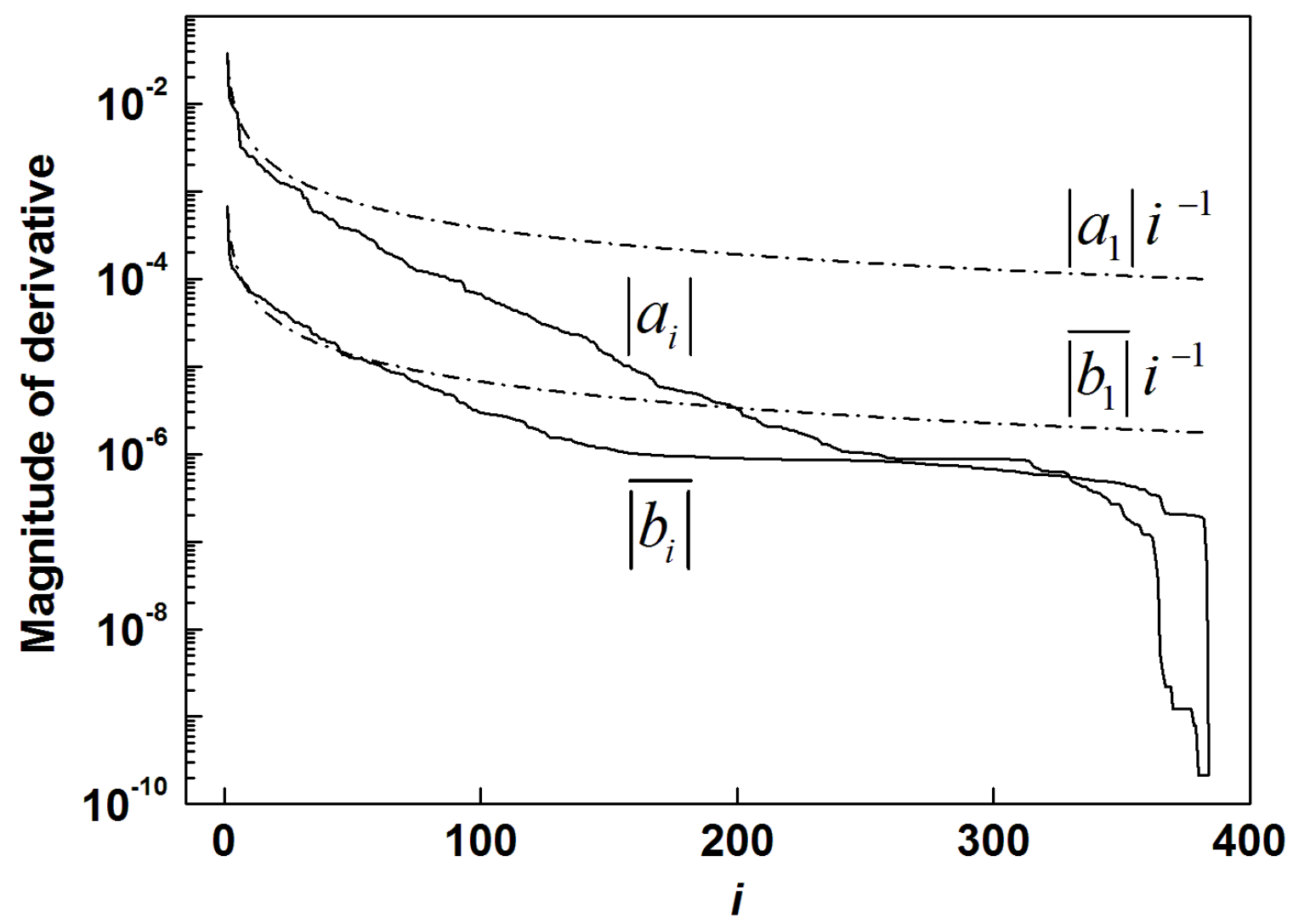

Figure 10. Decay rate of $\mathbf{a}_{\mathbf{r}}$ and $\mathbf{b}_{r}$ of eq. (9) (solid lines) calculated for the auto-ignition of $n$-butane at the pressure $1 \mathrm{~atm}$, initial temperature $1000 \mathrm{~K}$ and unity equivalence ratio. The $\mathbf{b}_{r}$ values are presented as the average $\left|\bar{b}_{i}\right|=1 / N_{r} \sum_{j=1}^{N_{r}}\left|b_{i j}\right| i$ is the reaction index. Dashed-dotted-dashed lines represent the series of reciprocals of positive integer numbers. 